\title{
Parasites of the Small Intestine
}

\author{
Theodore W. Schafer, MD, and Amer Skopic, DO
}

\section{Corresponding author}

Theodore W. Schafer, MD

Division of Gastroenterology, Naval Medical Center, 620 John Paul Jones Cir., Portsmouth, VA 23708, USA.

E-mail: twschafer@mar.med.navy.mil

Current Gastroenterology Reports 2006, 8:312-320

Current Science Inc. ISSN 1523-3790

Copyright (C) 2006 by Current Science Inc.

This paper discusses the most important parasites that inhabit the human small intestine. Beginning with the protozoa and proceeding through the various species of cestodes, nematodes, and trematodes that inhabit the human small bowel, the most important organisms are presented. Possible future developments are discussed along with pathophysiology and treatment in this phylogenic approach. Zoonotic illnesses, those diseases that by their rarity are of little import, and diseases without significant clinical or social implications are beyond the scope of this article and are not discussed.

\section{Introduction}

Parasites of the small intestine result in substantial morbidity and mortality worldwide. Areas of highest prevalence are those that can least afford the afflictions. Poverty, crowding, and the associated poor sanitation facilitate transmission of most of the organisms discussed. The consequences of malnutrition and attendant developmental delay are most prominent in children. Certain diseases present the opportunity for eradication or at least substantial control. Others will continue to afflict people for generations.

\section{Protozoa}

There are several important protozoan parasites of humans. Of these, only a few inhabit the small intestine and cause clinically important disease. The two most common small intestinal protozoan infections are caused by Giardia intestinalis and Cryptosporidium parvum. Treatment and other features of these parasites are found in Table 1.

\section{Giardiasis}

Giardia intestinalis (also called G. lamblia and G. duodenalis) is the most common intestinal parasite in the United States [1], with a rate of 7.4 infections per 100,000 population in 2002 [2•]. The organism has worldwide distribution.

Giardiasis is a clinical syndrome characterized by diarrhea, flatulence, and steatorrhea. The illness is typically mild to moderate but can range from asymptomatic to severe $[3,4]$.

Giardia intestinalis in the trophozoite form is a teardrop-shaped, binucleate eukaryotic organism with four pairs of flagella. The cyst form has four nuclei located at one pole of a roughly $8 \times 10-\mu \mathrm{m}$ densely walled structure. Diagnosis is by demonstration of the organism (typically cysts) in the feces or through a number of molecular methods. The most common diagnostic technique is stool antigen detection using enzyme-linked immunoassays. Sensitivities and specificities of various assays range from $88.6 \%$ to $100 \%$ and $99.3 \%$ to $100 \%$ [5].

The life cycle of $G$. intestinalis is relatively simple. The cyst form of the organism is ingested. After exposure to the low $\mathrm{pH}$ of the stomach and then the relatively neutral environment of the small bowel, the organism excysts into the trophozoite stage [6,7]. The trophozoite form can either divide by binary fission or encyst. The cysts are passed in the feces and are immediately infectious. Cysts can also survive in the environment for extended periods. The stimulus for cyst formation has not been elucidated.

In addition to environmental hardiness, Giardia organisms are also an important cause of zoonotic infection. A wide number of animal species, both domestic and wild, have been shown to harbor the parasite. Perhaps the best known animal reservoir for Giardia organisms is the beaver-hence the popular name for giardiasis: "beaver fever."

It is the trophozoite form of Giardia organisms dwelling in the small bowel, primarily the duodenum, that cause disease. The mechanism of disease is quite complex and not fully understood. Several different processes seem to occur simultaneously. The relative intensity and interplay of these mechanisms likely lead to the wide spectrum of disease seen in giardiasis. Key factors in the pathology of disease include villous atrophy, similar to that seen in celiac sprue [8], and impairment of carbohydrate digestion and absorption [9].

Treatment for giardiasis usually consists of metronidazole, $500 \mathrm{mg}$ orally three times a day for 5 days. Alternate therapies include albendazole and tinidazole [10]. As with all diseases transmitted by the fecal-oral 


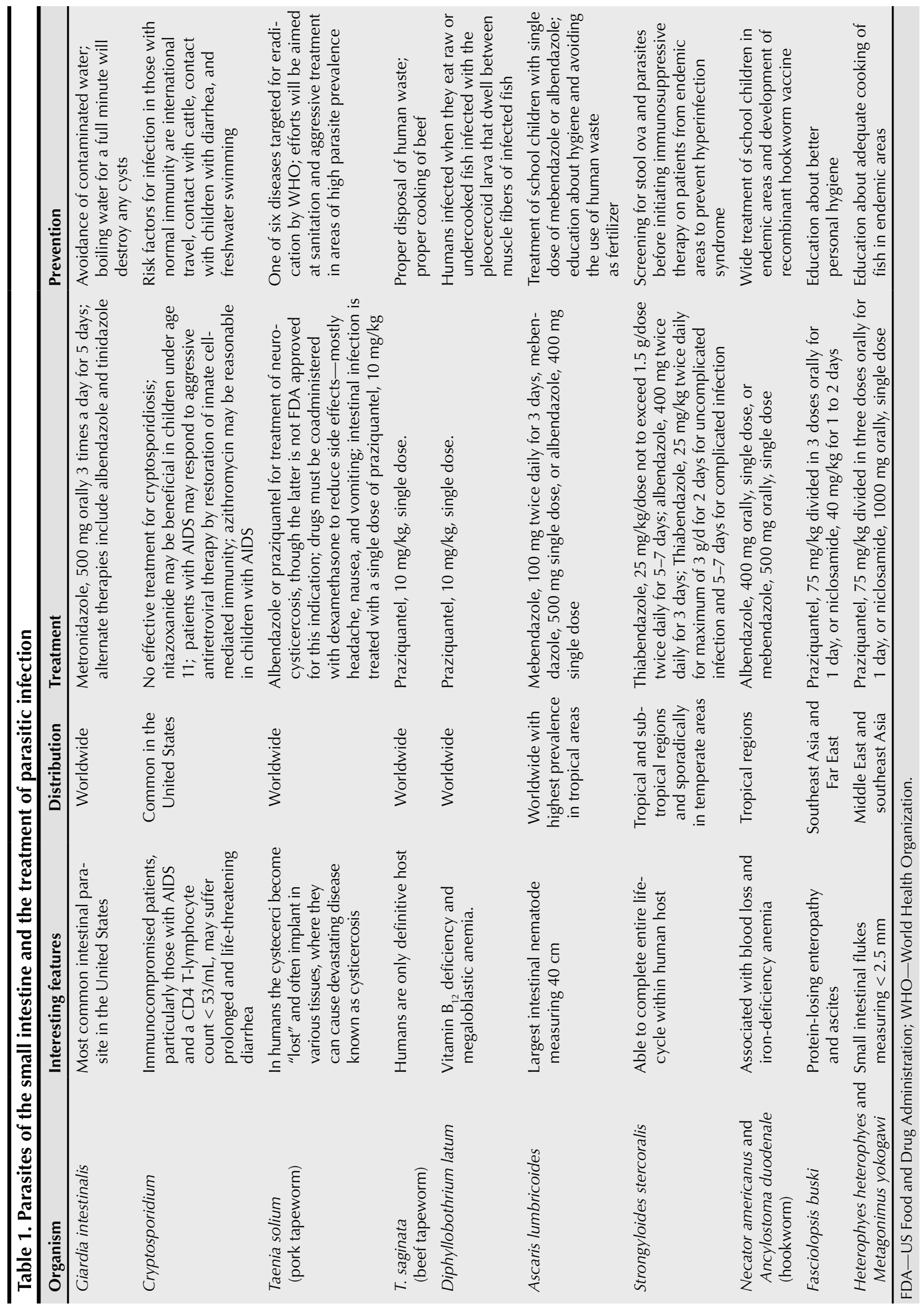


route, prevention is the best defense. Avoidance of contaminated water is the foundation for preventing transmission of giardiasis. In areas where a safe water supply cannot be guaranteed, boiling water for a full minute will destroy any cysts present.

\section{Cryptosporidiosis}

Cryptosporidiosis is another very common parasitic infection in the United States, with a rate of 1.0 per 100,000 population [2•]. As with giardiasis, infection with Cryptosporidium organisms causes diarrheal illness. However, the spectrum of disease is broader, ranging from asymptomatic to life threatening. Individuals with intact immunity typically suffer from watery diarrhea that lasts 3 to 14 days [11]. Abdominal cramping, low-grade fever, and malaise are typical symptoms. Risk factors for infection in those with normal immunity are international travel, contact with cattle, contact with children with diarrhea, and freshwater swimming [12].

Immunocompromised patients, particularly those with AIDS and a CD4 T-lymphocyte count of less than 53 per $\mathrm{mL}$, may suffer prolonged and life-threatening diarrhea [6,13]. In addition, people with AIDS are well known to be at risk for involvement of the biliary tract, with development of sclerosing cholangitis and the attendant problems associated with this illness $[7,14]$.

Two species of Cryptosporidium are important in humans, C. hominus (previously C. parvum genotype I) and C. parvum (previously C. parvum genotype II). Clinically, these organisms are indistinguishable. Transmission appears to be via the person-to-person [15] and zoonotic routes [16].

The life cycle of Cryptosporidium is quite complex and can take place entirely within a single host. Infection begins with ingestion of the oocyst form of the parasite. These oocysts release four sporozoites. The sporozoite form is internalized into the brush border of the gut epithelium, where it divides asexually to form merozoites. The merozoites are extruded into the gut lumen and infect more cells. Alternately, the merozoites may develop into gametocytes and combine to form oocysts that can either be shed into the environment or reinfect the host [17]. Thus, two opportunities exist within the life cycle for the parasite to autoinfect the host. This can result in severe and protracted infection, particularly in immunocompromised patients.

The diagnosis of cryptosporidiosis can be difficult, often requiring multiple stool samples to be established. With special staining techniques such as acid-fast and trichrome methods, the oocysts can be demonstrated in stool. Although it is simple to perform, this method relies on the experience of the observer and may not be reproducible between different laboratories. The most widely available methods use immunoassays for Cryptosporidium antigens in stool [18].

Currently, there is no effective treatment for cryptosporidiosis. Nitazoxanide may be of benefit in children aged under 11 years [19]. Patients with AIDS may respond to aggressive antiretroviral therapy by restoration of innate cell-mediated immunity [20]. Azithromycin may be a reasonable alternative, especially in the pediatric AIDS population [21,22].

\section{Helminths}

Intestinal parasitic worms, or helminths, infect millions of people worldwide. They represent a significant burden for countries where these parasites are endemic and cause significant morbidity and, potentially, mortality, especially in immunocompromised hosts.

Intestinal helminths are divided into three phyla based on general appearance and life cycle. The three major phyla of intestinal helminths are intestinal cestodes (tapeworms), nematodes (round worms), and trematodes (flukes). Treatment and other features of these infections can be found in Table 1 .

\section{Cestodes}

When considering parasites in the most general way, tapeworms are probably the first class of organisms that comes to mind. Although far more rare than some of the other organisms described in this paper, these parasites have thoroughly infected our literature and culture.

The life cycle of the various cestodes is complex. It depends on the close relationship of humans to various intermediate hosts to perpetuate the species. In general, the cycle begins when an infected individual passes fertile eggs in the stool. These eggs mature in the environment and are consumed by an intermediate host. In the intermediate host, the parasite infects the tissues (typically muscle) where it resides until this intermediate host is ingested by a human, the definitive host. Once the infected tissue is eaten, the parasite develops into an adult worm in the small intestine, completing the cycle. Adult worms may live for years and sometimes decades.

The tapeworms have many morphologic similarities as well. Each species has a distinct scolex, or attachment organ. Posterior to the scolex is a neck of variable size. The neck is the source of the fertile segments of the tapeworm known as proglottids. The bulk of most of the human tapeworms is the stroblia, or the entire collection of proglottids. The primary function of the proglottid is to serve as an "egg factory." Various worms shed the proglottids intact or release eggs directly into the intestinal lumen.

Tapeworm disease occurs under two circumstances. First, the adult worm itself usurps the nutrients of the host for its own use. Second, a human may become an inadvertent intermediate host, sometimes with devastating consequences.

\section{Taeniasis}

Two species of the genus Taenia are important in humans. $T$. saginata, the beef tapeworm, and T. solium, the pork tapeworm, are distributed worldwide. The close association 
of humans to the intermediate host animals assures perpetuation of these organisms. The Centers for Disease Control estimate that 50 million persons are infected with either T. solium or T. saginata worldwide [23•].

The life cycle of $T$. saginata follows the general scheme outlined previously. Eggs from an infected host contaminate the soil or vegetation eaten by cattle, the intermediate host. The eggs hatch into oncospheres that penetrate the intestinal wall and ultimately migrate to muscle tissues of the bovine intermediary. In the muscle tissue the oncospheres develop into cystecerci. The cystecerci can remain in place for many years. For the cycle to continue, undercooked or raw beef must be eaten by humans because cooking kills the cystecerci. After 10 to 12 weeks the worm is fully developed and typically measures about $5 \mathrm{~m}$ in length. The worm is folded back on itself and inhabits the small intestine. Symptoms of infection with adult worms are infrequent and mild. Perhaps the most troubling symptom is passage of intact proglottids or long segments of the stroblia.

Infection with the pork tapeworm, T. solium, is essentially the same as with the beef tapeworm. The key difference is the intermediate host, which is swine instead of cattle. Adult worms are up to $7 \mathrm{~m}$ long. T. solium has one other aspect that is much more sinister. Unlike the beef tapeworm, the fertile eggs of the pork tapeworm are infective to humans. When ingested, the eggs attempt to develop as they would in swine. However, in humans the cystecerci become "lost" and often implant in various tissues, where they can cause a devastating disease known as cysticercosis. The most serious forms of human cysticercosis occur when the parasite inhabits the central nervous system.

Neurocysticercosis is the most common cause of seizures in areas where the disease is endemic [24]. Risk factors for high rates of cysticercosis include poor sanitary conditions and lack of pork inspection prior to marketing [24,25]. Seizures frequently recur [26]. Nearly 50,000 deaths occur worldwide each year due to cysticercosis [23•].

Rates of cysticercosis are difficult to determine for a variety of reasons, including poor or absent reporting in developing nations [27] and transient positive serology in non-infected people [28]. However, in some rural areas of Central and South America, rates of neurocysticercosis exceed $20 \%$. Rates approaching $3 \%$ have been noted in migrant farm workers in southern California [29].

Diagnosis can be made though various immunoassays of either serum or cerebrospinal fluid. Electroimmunotransfer blot assay using purified T. solium antigens is the most specific method, but enzyme-linked immunosorbent assay is less expensive to perform and thus probably better suited to epidemiologic studies [30]. Imaging with either MRI or CT is also diagnostic [31].

Treatment of neurocysticercosis is with albendazole or praziquantel, though the latter is not approved by the US Food and Drug Administration (FDA) for this indication
[32]. Unfortunately, neither drug is completely or uniformly effective [33]. The drugs must be coadministered with dexamethasone to reduce side effects-mostly headache, nausea, and vomiting. Deaths during treatment, usually from stroke, have been reported. There is no significant reduction in seizures after treatment. Despite these limitations, most authorities recommend anti-cysticercal therapy, though the issue remains controversial [34-36].

Because the pork tapeworm has only one definitive host-humans-it is one of the six diseases targeted for eradication by the World Health Organization [23•]. Efforts will be aimed at sanitation and aggressive treatment in areas of high parasite prevalence.

\section{Fish tapeworm}

Diphyllobothrium latum is another tapeworm with worldwide distribution. This parasite has two different intermediate hosts: several species of copepod and subsequently any one of a number of freshwater vertebrate fish. Humans are infected when they eat raw or undercooked fish infected with the pleocercoid larva that dwell between muscle fibers of infected fish. Overt symptoms are uncommon and are similar to those of taeniasis. Adult worms can exceed $12 \mathrm{~m}$ in length and shed tens of millions of eggs daily.

Most interesting is the association of D. latum with vitamin $B_{12}$ deficiency and megaloblastic anemia. Vitamin $\mathrm{B}_{12}$ absorption takes place exclusively in the terminal ileum. In infected persons, therefore, the parasite gets exposed to the vitamin $B_{12}$ prior to the host being able to absorb it [37]. Why this does not occur with other parasites of the small bowel is unknown. Treatment is with praziquantel.

\section{Nematodes}

Nematodes share a common life cycle. They infect people via multiple routes, including fecal-oral, direct contact with infected soil or water, and ingestion of infected fish and meat. After the egg or larval stage enters the body, the parasite directly enters the vasculature, migrates to the lungs, and crosses from the capillaries into alveolar spaces. Next, the parasite ascends the airway to the pharynx. The worms are then swallowed. In the small intestine the larvae mature into adults and become fertile, laying eggs into to the bowel lumen. These eggs are expelled in the feces, completing the cycle. Some species can complete the entire cycle within a single host, whereas others require either an intermediate host or soil larval stage.

\section{Ascariasis}

Ascaris lumbricoides is the largest human intestinal nematode and can reach $40 \mathrm{~cm}$ in length. Nearly a fourth of the world's population, 1.4 billion people, are infected. Ascaris infection causes 20,000 deaths a year worldwide [38]. Factors contributing to the high incidence of Ascaris infection are the ability to produce up to 200,000 eggs daily that survive for extended 
periods in soil, poor socioeconomic conditions, the generally asymptomatic nature of the infection, and lack of protective immunity [39].

A. lumbricoides is transmitted through ingestion of fecally contaminated food. The Ascaris life cycle begins by passage of fertile ova in stool and follows the general nematode cycle already described. It takes about 3 months from infection until the worms produce eggs [40]. The larval form is capable of crossing the placenta and infecting the unborn fetus. The exact rates of stillbirths due to fetal infection are not known [41].

Adult worms are typically found in the jejunum or ileum, where they can survive for 2 years. The worm is sexually dimorphic, thus requiring presence of both genders in the intestinal tract to produce viable eggs. Diagnosis of $A$. lumbricoides infection is based on demonstrated presence of eggs in stool.

Endemic areas of HIV and Ascaris infection overlap in multiple regions, and it is important to define the relationship between these two infections. HIV enteropathy and related changes in the immune response of the intestinal epithelium appear to provide protection against luminal nematodes, resulting in lower prevalence of ascariasis in HIV-positive individuals [42]. In contrast, this same study showed that the rate of intracellular infections, such as cryptosporidiosis, was much higher in HIV-positive individuals.

Clinical disease is directly related to parasite burden. This is a function of continuous exposure to infectious second-stage larvae [43]. Clinical symptoms are caused by either tissue reactions with damage due to migrating larvae or by the presence of heavy adult parasite burden of worms in the small intestine.

Recurrent ascariasis in children can cause stunted linear growth, reduced cognitive function, and malnutrition [44]. Children infected with $A$. lumbricoides are found to have decreased absorption of vitamins $\mathrm{A}$ and $\mathrm{C}$ and lower serum albumin than uninfected children [45]. With treatment, many children are able to regain lost growth. Factors in determining whether a child with stunted growth is able to "catch up" after treatment are the extent of the growth deficit, appropriate nutrition, and duration of the diseasefree state [46].

Larval migration can result in Loeffler's syndrome, which occurs 1 to 2 weeks after infection. Symptoms of eosinophilic pneumonitis, including pleuritic chest pain, dyspnea, wheezing, fever, and occasionally hemoptysis, should raise suspicion in the treatment of susceptible patients. The chest radiograph may show bilateral infiltrates, which are migratory and usually clear spontaneously and completely after several weeks [47].

Gastrointestinal complications caused by a heavy parasite load include obstruction, intussusception, perforation, and appendicitis. Worms occasionally invade the biliopancreatic tract. Consequences include biliary colic, acute cholecystitis, acute cholangitis, acute pancreatitis. and hepatic abscess [38]. Patients with one episode of
Ascaris-induced biliary tract invasion may be at risk for subsequent episodes [48]. This raises the question of host factors attracting the parasites to unusual locations.

Treatment is with albendazole or mebendazole. Individuals should be followed for 3 to 4 months after treatment to ensure that the worms have been eliminated. Reinfection in endemic areas exceeds $80 \%$ within 6 months [38]. Mass treatment of all school children with a single dose of albendazole or mebendazole every 3 to 4 months in endemic areas has decreased intensity of the infection without effect on prevalence [49].

\section{Strongyloidiasis}

Strongyloides stercoralis is the only nematode that is able to complete its entire life cycle within one human host. Individuals with HIV infection have increased risk for superinfection and disseminating disease. This parasite is endemic in tropical and subtropical regions and occurs sporadically in temperate areas. Estimated worldwide prevalence is about 60 million infections. In the United States, the highest rates of infection are found in immigrants from endemic areas, mental institutions, and immunosuppressed persons. The highest prevalence in the United States is in Appalachia and can be as high as $4 \%$ [40].

The life cycle is similar to that of other nematodes with important exceptions. Strongyloides organisms reside in the duodenum and proximal jejunum. There they burrow into intestinal mucosa and transform into the hermaphroditic adult. After 3 weeks, adults produce eggs that may develop into noninfectious larvae (rhabditiform larvae), which are usually passed in feces. These larvae can metamorphose into the filariform larvae, which can reinfect the host by invading bowel wall (prior to being passed) or perianal skin [40]. These organisms are relatively small in size, measuring 2 to $2.5 \mathrm{~mm} \times 30$ to $50 \mu \mathrm{m}$.

S. stercoralis organisms live in the human small intestine up to 5 years by downregulating the immune system of the human host. This effect is mediated by the ability to decrease numbers of mature macrophages, resulting in the lack of immune response demonstrated by similar profiles of intraepithelial lymphocytes in small intestine in infected patients and control subjects [50].

Clinical symptoms are usually mild and consist of nonspecific gastrointestinal, cutaneous, or pulmonary symptoms that persist for years. Eosinophilia can occur in the absence of symptoms, and up to $30 \%$ of infected people are asymptomatic [51]. Strongyloides infection may also occasionally be suspected during an upper gastrointestinal series due to the reflux of barium into the biliary tract caused by an incompetent sphincter of Oddi. This sphincter incompetence is caused by the rigidity of the duodenal wall in the situation of chronic scarring from the invasive worm [52].

In contrast, the potentially fatal hyperinfection syndrome has multiorgan manifestations. The symptoms of hyperinfection include fever, chills, and anorexia. The 
skin may demonstrate a non-blanching, serpiginous, petechial rash that suggests invasion from cutaneous venous circulation [52-54]. The most common gastrointestinal symptoms are generalized abdominal pain, nausea, vomiting, and bloody and non-bloody diarrhea. Complications of pulmonary involvement can result in adult respiratory distress syndrome (ARDS) and respiratory failure in $50 \%$ of cases. Strongyloides organisms carry bacteria across the intestinal wall into the blood as they migrate, resulting in sepsis, meningitis, peritonitis, and endocarditis. These secondary infections are associated with high mortality [55-57].

Hyperinfection syndrome begins with autoinfection within the gastrointestinal tract of the host. When rhabditiform larvae mature into filariform larvae, they penetrate the intestinal wall and enter the bloodstream. In the hyperinfection syndrome, autoinfection is significantly increased, with a markedly elevated worm burden. Filariform larvae can disseminate to multiple organs and cause severe local inflammation reactions, as described previously.

Patients at risk for disseminated infections include those with lymphoproliferative malignancies, those being treated with chemotherapy or steroids, and HIVor human T-cell lymphocytic virus (HTLV-1)-positive individuals. Infection with HTLV-1 is a greater risk for hyperinfection than HIV-1 infection, most likely due to suppression of IgE [54-57]. Intermittent or continuous use of corticosteroids is another common risk. Screening with stool ova and parasites for Strongyloides organisms should be done before starting immunosuppressive therapy in individuals from endemic areas due to the risk of hyperinfection syndrome [58].

The diagnosis is based on the clinical syndrome of gastrointestinal and skin findings and testing of stool for ova and parasites. However, about $25 \%$ of infected patients have negative results in stool examinations [58]. The Baerman concentration technique and modified agar plate method can increase sensitivities of stool studies. Aspirated duodenojejunal fluid and Enterotest (HDL Corp., Huntington Beach, CA) have approximately $90 \%$ sensitivity [51,58]. The most accurate method of diagnosis is the enzyme-linked immunoabsorbent assay (ELISA), which is highly sensitive and specific, even in cases of repeatedly negative results from stool studies [54].

Both thiabendazole and ivermectin are effective treatments. Ivermectin is better tolerated by most individuals. Patients need to be closely followed after treatment because relapses and treatment failures are not uncommon.

\section{Hookworm}

Hookworm disease, caused by infection with Necator americanus and Ancylostoma duodenale organisms, is the most common cause of chronic intestinal infection and accounts for an estimated 740 million cases in the tropics. Hookworm is mostly found in areas with high rainfall [59]. Hookworm was almost eliminated from the
United States after extensive control efforts, although it was endemic in the southeastern United States at the turn of the 20th century [60].

The life cycle of hookworm infection begins with thirdstage larvae that live in the soil. When the larva comes in contact with human skin, it penetrates almost instantly. It then follows the general life cycle of nematodes, as discussed earlier. When the eggs are expelled they develop in the soil and wait for the next passer-by.

Hookworm infections cause intestinal blood loss and consequent iron-deficiency anemia $[59,60]$. Hookworm is able to cause chemical and mechanical breakdown of small arterioles and veins in the intestinal wall. The organism applies its mouth, which serves as a suction cap, to the intestinal surface and induces mechanical rupture of the mucosal blood vessels. Hookworms also produce hydrolytic enzymes, which chemically break down the vessel wall [59]. In addition, these worms secrete anticoagulants that are now being investigated for the treatment of thrombophilia associated with Ebola virus infection [61].

The clinical presentation of chronic hookworm infection includes symptomatic anemia, recurrent epigastric pain, nausea, exertional dyspnea, palpitations, fatigue, and headache $[62,63]$. The severity of clinical presentation and anemia is directly related to worm burden. Hemoglobin levels of less than $11 \mathrm{~g} / \mathrm{dL}$ are associated with a hookworm burden of approximately 100. A. duodenale may also cause a syndrome called Wakana disease, which is characterized by nausea, vomiting, pharyngeal irritation, and cough [64].

A laboratory finding associated with hookworm infection is eosinophilia, which is usually detected 5 to 9 weeks after onset of infection [60]. Hookworm infection may also cause hypoalbuminemia from decreased absorption in the intestinal tract, presenting as diffuse anasarca and ascites in severe infections. Hookworm infection is diagnosed by demonstration of eggs in the stool. Adult worms can be detected endoscopically in symptomatic individuals [64].

Children are most vulnerable to hookworm infectionrelated consequences. Hookworm infections are directly related to stunted physical growth and diminished memory, reasoning ability, and reading comprehension in children $[65,66]$. In contrast to other helminthic infections, however, in which worm burden peaks in childhood, hookworm infections tend to peak or plateau in adulthood [67]. These observations led to theories that hookworm infections can either evade or suppress the host immune system $[68,69 \bullet]$.

Immunomodulatory molecules isolated from the adult hookworm include T-cell apoptotic factor, an integrin antagonist of host CD11b and CD18, retinol-binding protein, a C-type lectin, a tissue inhibitor of metaloproteases, cysteinerich secretory proteins, and an eotaxin-degrading factor [69•]. From these data it can be reasoned that hookworm infection predisposes infected individuals to other infections. Because hookworm infection is one of the most common 
chronic intestinal infections worldwide, the consequence of "secondary" infections, particularly susceptibility to HIV, malaria, and tuberculosis, is currently being explored [70-72]. Two preliminary studies have failed to support this concept, however [73,74]. Further study is ongoing.

The only FDA-approved treatment of hookworm is with mebendazole for 3 days, $100 \mathrm{mg}$ orally twice a day (an alternate dose of $500 \mathrm{mg}$ in 1 day has been used). Alternative medications include pyrantel pamoate and albendazole. The World Health Organization has set a goal to provide routine treatment for at least $75 \%$ of all school-age children at risk by 2010 [75]. The aim of this intervention is to minimize the impact of hookworm on physical and neurologic development. The added benefit of reducing worm burden may be to reduce other infections.

The Sabin Vaccine Institute, in partnership with the Department of Microbiology and Tropical Medicine of The George Washington University Laboratory, has developed a genetically engineered recombinant hookworm vaccine. Clinical trials to test the safety of the hookworm vaccine are anticipated to start in 2005. Hookworm vaccine is designed as a bivalent vaccine. A part of the vaccine is derived from third-stage larvae to reduce hookworm burden, and the other part is derived from adult hookworm to decrease blood loss. The vaccine is designed to be used in combination with antihelminthic agents. Advantages of the vaccine are decreased reliance on antihelminthics, better results in areas where traditional methods have failed, and avoidance or reduction of drug-resistant strains of hookworm [76]. US phase I and phase II trials in endemic areas of Brazil are underway. Results are expected to be reported in 2007 and will lay the groundwork for larger clinical trials to evaluate the efficacy and safety of this vaccine [76].

\section{Trematodes}

Fasciolopsis buski is a large intestinal fluke (measuring up to $75 \mathrm{~mm}$ in length) that is a common parasite of pigs and humans in southeast Asia and the Far East. Two other small intestinal trematodes, Heterophyes heterophyes and Metagonimus yokogawi, measure less than $2.5 \mathrm{~mm}$ in length. $H$. heterophyes is found predominantly in the Middle East. M. yokogawi is endemic in eastern Asia [77-79].

Humans acquire infection by ingestion of contaminated water or plants (F. buski) or by eating raw fish (H. heterophyes and M. yokogawi). Eggs, being passed into fresh water from inappropriate disposal of waste, mature over a few weeks into free-swimming miracidia. Miracidia develop into cercariae in the tissue of snails over a period of 3 to 5 months. After the cercariae are released from the snail back into the water, they encyst on the leaves of any one of a number of common food plants (F. buski). $H$. heterophyes and $M$. yokogawi cercariae, however, encyst in the tissues of certain freshwater fish. When the contaminated fish are consumed uncooked, humans become infected. Adult flukes survive for approximately 1 year. They are usually found in the duodenum and jejunum.
F. buski infection is usually asymptomatic. Symptoms, when present, occur from a heavy parasite load. Typical symptoms include anorexia, nausea, vomiting, and malabsorption, which can result in severe protein loss that can manifest with edema and ascites [77,78].

Symptoms of $H$. heterophyes and M. yokogawi infection are associated with necrosis and ulceration of the intestinal mucosa. Anorexia, nausea, abdominal pain, weight loss, dyspepsia, and mucous diarrhea may occur with an especially high parasite burden $[78,79]$. Occasionally, eggs spread hematogenously to ectopic sites such as the heart or central nervous system [78].

Diagnosis of intestinal fluke infection is made by demonstration of eggs or adult worms in the stool. Diagnostic yield can be increased by concentration of stool. Treatment is with praziquantel in three divided doses for 1 day. Follow-up stool studies should be performed to confirm eradication.

\section{Conclusions}

The majority of the world's population harbors at least one parasite of the small intestine. Most of these infections are subclinical. Despite the absence of symptoms, however, the impact in developing nations is profound. Physical and neurologic development may be delayed or stunted. Infected persons are at risk for other diseases. Most of these illnesses can be controlled-and in some cases eliminated-through improved sanitation and targeted treatment. At present, vaccines do not prevent illness entirely but serve as an adjunct to standard medications.

\section{Disclaimer}

The views expressed in this article are those of the authors and do not reflect the official policy or position of the Department of the Navy, Department of Defense, or the United States Government.

\section{References and Recommended Reading}

Papers of particular interest, published recently, have been highlighted as:

- Of importance

-. Of major importance

1. Hanson KL, Cartwright CP: Use of an enzyme immunoassay does not eliminate the need to analyze multiple stool specimens for sensitive detection of Giardia lamblia. J Clin Microbiol 2001, 39:474-477.

2. Centers for Disease Control and Prevention: Cryptosporidiosis Surveillance - United States 1999-2002 and Giardiasis Surveillance - United States, 1998-2002. In Surveillance Summaries, January 28,2005. MMWR 2005:54(No. SS-1). This is a valuable overview of the impact, transmission, and control of these two important parasitic illnesses. Its value is underscored by its timeliness and relevance to a broad spectrum of medical practices.

3. Thompson RC: Giardiasis as a re-emerging infectious disease and its zoonotic potential. Int J Parasitol 2000, 30:1259-1267. 
4. Kappus KD, Lundgren RG, Juranek, DD, et al.: Intestinal parasitism in the United States: update on a continuing problem. Am J Trop Med Hyg 1994, 50:705-713.

5. Aldeen WE, Carroll K, Robison A, et al.: Comparison of nine commercially available enzyme-linked immunosorbent assays for detection of Giardia lamblia in fecal specimens. J Clin Microbiol. 1998, 36:1338-1340.

6. Bingham AK, Meyer EA: Giardia excystation can be induced in vitro in acidic solutions. Nature 1979, 277:301-302.

7. Boucher SEM, Gillin FD: Excystation of in vitro-derived Giardia lamblia cysts. Infect Immun 1990, 58:3516-3522.

8. Mastropasqua E, Rolandi R, Farruggio A, Lirussi F: Giardia duodenalis: a confounding factor for the diagnosis of celiac disease. J Clin Gastroenterol 2003, 36:185-186.

9. Hartong WA, Gourley WK, Arvanitakis C: Giardiasis: clinical spectrum and functional-structural abnormalities of the small intestinal mucosa. Gastroenterology 1979, 77:61-69.

10. Farthing MJ. The molecular pathogenesis of giardiasis. J Pediatr Gastroenterol Nutr 1997, 24:79-88.

11. Rossignol JA, Ayoub A, Ayers MS: Treatment of diarrhea caused by Cryptosporidium parvum: a prospective randomized, double-blind, placebo-controlled study of nitazoxanide. J Infect Dis 2001, 184:103-106.

12. Roy SL, DeLong SM, Stenzel SA, et al.: Emerging Infections Program FoodNet Working Group. Risk factors for sporadic cryptosporidiosis among immunocompetent persons in the United States from 1999 to 2001. J Clin Microbiol 2004 42:2944-2951.

13. Colford JM Jr, Tager IB, Hirozawa AM, et al.: Cryptosporidiosis among patients infected with human immunodeficiency virus. Factors related to symptomatic infection and survival. Am J Epidemiol 1996, 144:807-816.

14. Goldin RD, Hunt J: Biliary tract pathology in patients with AIDS. J Clin Pathol 1993, 46(8):691-693.

15. Tumwine JK, Kekitiinwa A, Nabukeera N, et al.: Cryptosporidium parvum in children with diarrhea in Mulago Hospital, Kampala, Uganda. Am J Trop Med Hyg 2003, 68(6):710-715.

16. Smerdon WJ, Nichols T, Chalmers RM, et al.: Foot and mouth disease in livestock and reduced cryptosporidiosis in humans, England and Wales. Emerg Infect Dis 2003, 9:22-28.

17. Chen XM, Keithly JS, Paya CV, LaRusso NF: Cryptosporidiosis. N Engl J Med 2002, 346:1723-1731.

18. Mehta P: Laboratory diagnosis of cryptosporidiosis. J Postgrad Med 2002, 48:217-221.

19. Rossignol JF, Ayoub A, Ayers MS: Treatment of diarrhea caused by Cryptosporidium parvum: a prospective randomized, double-blind, placebo-controlled study of nitazoxanide. J Infect Dis 2001, 184:103-106.

20. Miao YM, Awad-El-Kariem FM, Franzen C, et al.: Eradication of cryptosporidia and microsporidia following successful antiretroviral therapy. J Acquir Immune Defic Syndr 2000, 25:124-129.

21. Ramos JT, Saavedra J, Ruiz-Contreras J: Cryptosporidium in patients infected with human immunodeficiency virus: azithromycin revisited. J Pediatr 1997, 130:1009-1010.

22. Dionisio D, Orsi A, Sterrantino G, et al.: Chronic cryptosporidiosis in patients with AIDS: stable remission and possible eradication after long-term, low dose azithromycin. J Clin Pathol 1998, 51:138-142.

23. Centers for Disease Control and Prevention: Recommendations of the International Task Force for Disease Eradication. MMWR 1993, 42(No. RR-16).

This reference provides valuable insight into global population medicine. It includes summaries of all six diseases slated for eradication and also several others that may be future targets for eradication.

24. Garcia HH, Gilman RH, Gonzalez AE, et al.: Cysticercosis Working Group in Peru. Hyperendemic human and porcine Taenia solium infection in Peru. Am J Trop Med Hyg 2003, 68:268-275.
25. Vazquez-Flores S, Ballesteros-Rodea G, Flisser A, Schantz PM: Hygiene and restraint of pigs is associated with absence of Taenia solium cysticercosis in a rural community of Mexico. Salud Publica Mex 2001, 43:574-576.

26. Carpio A, Hauser WA: Prognosis for seizure recurrence in patients with newly diagnosed neurocysticercosis. Neurology 2002, 59:1730-1734.

27. Bragazza LM, Vaz AJ, Passos AD, et al.: Frequency of serum anti-cysticercus antibodies in the population of a rural Brazilian community (Cassia dos coqueiros, SP) determined by ELISA and immunoblotting using Taenia crassiceps antigens. Rev Inst Med Trop Sao Paulo 2002, 44:7-12.

28. Garcia HH. Gonzalez AE. Gilman RH. et al.: Cysticercosis Working Group in Peru. Short report: transient antibody response in Taenia solium infection in field conditions-a major contributor to high seroprevalence. Am J Trop Med Hyg 2001, 65:31-32.

29. DeGiorgio C, Pietsch-Escueta S, Tsang V, et al.: Seroprevalence of Taenia solium cysticercosis and Taenia solium taeniasis in California, USA. Acta Neurol Scand 2005, 111:84-88.

30. Proano-Narvaez JV, Meza-Lucas A, Mata-Ruiz O, et al.: Laboratory diagnosis of human neurocysticercosis: doubleblind comparison of enzyme-linked immunosorbent assay and electroimmunotransfer blot assay J Clin Microbiol 2002, 40:2115-2118.

31. Suss RA, Maravilla KR, Thompson J: MR imaging of intracranial cysticercosis: comparison with $\mathrm{CT}$ and anatomopathologic features. Am J Neuroradiol 1986, $7: 235-242$.

32. Mehta SS, Hatfield S, Jessen L, Vogel D: Albendazole versus praziquantel for neurocysticercosis. Am J Health Syst Pharm 1998, 55:598-600.

33. Garcia HH, Gilman RH, Horton J, et al.: Albendazole therapy for neurocysticercosis: a prospective double-blind trial comparing 7 versus 14 days of treatment. Cysticercosis Working Group in Peru. Neurology 1997, 48:1421-1427.

34. Hachinski V: Medical treatment of cysticercosis. Arch Neurol 1995, 52:104.

35. del Brutto $\mathrm{OH}$ : Medical treatment of cysticercosis-effective. Arch Neurol 1995, 52:102-104.

36. Kramer LD: Medical treatment of cysticercosis-ineffective. Arch Neurol 1995, 52:101-102.

37. von Bonsdorff B, Gordin R: Castle's test (with vitamin $\mathrm{B} 12$ and normal gastric juice) in the ileum in patients with genuine and patients with tapeworm pernicious anaemia. Acta Med Scand 1980, 208:193-197.

38. Khuroo MS: Asciriasis. Gastroenterol Clin North Am 1996, 25:553-577.

39. Seltzer, E. Ascariasis. In Tropical Infectious Diseases: Principles, Pathogens and Practice, edn 1. Edited by Guerrant RL, Weller PF. Philadelphia: Churchill Livingstone; 1999:553-577.

40. Mahmoud AAF: Intestinal nematodes (roundworms). In Principles and Practice of Infectious Diseases, edn 5. Edited by Mandell GL, Douglas RG, Benett JE. New York: Churchhill Livingstone; 2000:2938-2943.

41. Chu, WG, Chen PM, Huag CC et al.: Neonatal ascariasis. J Pedriatr 1972, 81:783-785.

42. Lindo JF, Dubon JM, Ager AL, et al.: Intestinal parasitic infections in human immunodeficiency virus ( HIV )-positive and HIV-negative individuals in San Pedro Sula, Honduras. Am J Trop Med Hyg 1998, 58:431-435.

43. Swartzman J: Ascariasis. In Hunter's Tropical Medicine and Emerging Infectious Disease, edn 8. Edited by Strickland GT. Philadelphia: WB Saunders Co.; 2000:726-729.

44. Oberhelmon RA, Guerrero ES, Fernandez ML, et al.: Correlation between intestinal parasitosis, physical growth, and psychomotor development among infants and children from rural Nicaragua. Am J Trop Med Hyg 1998, 58:470-475.

45. Hlaing T: Ascariasis and childhood malnutrition. Parasitology 1993, 107(Suppl):S125-S136. 
46. Hall A: Intestinal parasitic worms and the growth of children. Trans R Soc Trop Med Hyg 1993, 87:241-242.

47. Weller, PF: Parasitic pneumonias. In Respiratory Infections: Diagnosis and Management, edn 3. Edited by Pennington JE. New York: Raven Press; 1994:695.

48. Shad JA, Lee YR: Pancreatits due to Ascaris lumbricoides: second occurance after 2 years. South Med J 2001, 94(1):78-80.

49. Hall A, Anwar KS, Tomkins AM: Intensity of reinfection with Ascaris lumbricoides and its implications for parasite control. Lancet 1992, 339:1253-1257.

50. Igra-Siegman Y, Kapila R, Sen P, et al.: Syndrome of hyperinfection with Strongyloides stercoralis. Rev Infect Dis 1981, 3:397-407.

51. Beal CB, Viens P, Grant RG, Hughes JM: A new technique for sampling duodenal contents. Demonstration of upper smallbowel pathogens. Am J Trop Med Hyg 1970, 19:349-252.

52. Savage D, Foadi M, Haworth C, Grant A: Marked eosinophiliain an immunosuppresed patient with strongyloidiasis. J Intern Med 1994, 236:473-475.

53. Kalg RE, Grossman ME: Periumbilical purpura in disseminated strongyloidiasis. JAMA 1986, 256:1170-1171.

54. Scowden EB, Schaffner W, Stone WJ: Overwhelming strongyloidiasis: an unappreciated opportunistic infection. Medicine (Baltimore) 1978, 57:527-544.

55. Genta RM: Global prevalence of strongyloides: critical review with epidemiological insights into the prevention of disseminated disease. Rev Infect Dis 1989, 11:755-767.

56. Linder JD, Monkemuller KE, Lazenby AJ, Wilcox CM: Streptococcus bovis bacteremia associated with Strongyloides stercoralis colitis. Gasrointest Endosc 2000, 52:796-798.

57. Lee MG, Terry SI: Arteriomesenteric duodenal occlusion associated with strongyloidiasis. J Trop Med Hyg 1989, 92:41-45.

58. Sato Y, Kobayashi J, Toma H, Shiroma Y: Efficacy of stool examination for detection of Strongyloides infection. $\mathrm{Am} \mathrm{J}$ Trop Med Hyg 1995, 539:248-250.

59. Maxwell C, Hussain R, Nutman TB, et al.: The clinical and immunologic responses of normal human volunteers to low dose hookworm (Necator americanus) infection. Am J Trop Med Hyg 1987, 37:126-134.

60. Hotez PJ, Pritchard DI: Hookworm infection. Sci Am 1995, 272:68-74.

61. Stanssens P, Bergum PW, Gansemans Y, et al.: Anticoagulant repertoire of the hookworm Ancylostoma caninum. Proc Natl Acad Sci USA 1996, 93:2149-2154.

62. Anyaeze CM: Reducing burden of hookworm disease in the management of upper abdominal pain in the tropics. Trop Doct 2003, 33:174-175.

63. de Silva, NR, Brooker, S, Hotez, PJ, et al.: Soil-transmitted helminth infections: updating the global picture. Trends Parasitol 2003, 19:547-551.

64. Kato T, Kamoi R, Iida M, et al.: Endoscopic diagnosis of hookworm disease of the duodenum. J Clin Gastroenterol 1997, 24:100-102.
65. Stephenson LS, Latham MC, Kurz KM, et al.: Treatment with a single dose of albendazole improves growth of Kenyan schoolchildren with hookworm, Trichuris trichiura, and Ascaris lumbricoides infection. Am J Trop Med Hyg 1989, 41:78-87.

66. Sakti H, Nokes C, Hertanto WS, et al.: Evidence for an association between hookworm infection and cognitive function in Indonesian school children. Trop Med Int Health 1999, 4:322-334.

67. Bethony J, Chen J, Lin S, et al.: Emerging patterns of hookworm infection: influence of aging on the intensity of Necator infection in Hainan Province, People's Republic of China. Clin Infect Dis 2002, 35:1336-1344.

68. Olatunde BO, Onyemelukwe GC, et al.: Immunosuppression in Nigerians with hookworm infection. Afr J Med Sci 1994, 23:221-225.

69. Loukas A, Prociv P: Immune responses in hookworm infections. Clin Microbiol Rev 2001, 14:689-703.

The authors provide an interesting insight into future directions for reducing the toll of hookworm infection throughout the world.

70. Brooker S, Bethony J, Hotez PJ: Human hookworm infection in the 21st century. Adv Parasitol 2004, 58:197-288.

71. Fincham JE, Markus MB, Adams VJ: Could control of soiltransmitted heminthic infection influence the HIV/AIDS pandemic? Acta Trop 2003, 86:315-333.

72. Nacher M, Singhasivanon P, Yimsamran S, et al.: Intestinal helminth infections are associated with increased incidence of Plasmodium falciparum malaria in Thailand. $J$ Parasitol 2002, 88:55-58.

73. Shapiro AE, Tukahebwa EM, Kasten J, et al.: Epidemiology of helminth infections and their relationship to clinical malaria in southwest Uganda. Trans R Soc Trop Med Hyg 2005, 99:18-24.

74. Modjarrad K, Zulu I, Redden DT, et al.: Treatment of intestinal helminths does not reduce plasma concentrations of HIV-1 RNA in coinfected Zambian adults. J Infect Dis 2005, 192:1277-1283.

75. Prevention and control schistosomiasis and soil-transmitted helminthiasis. World Health Organ Tech Rep Ser 2002, 912:1-57.

76. Sabine Vaccine Institute: Human hookworm vaccine initiative (HHVI). Available at: http://www.sabin.org/hookworm.htm. Accessed 2/26/05.

77. Chai JY, Lee SH: Food-borne intestinal trematode infections in the Republic of Korea. Parasitol Int 2002, 51:129-154.

78. Liu, LX, Harinasuta, KT: Liver and intestinal flukes. Gastroenterol Clin North Am 1996, 25:627-636.

79. Belizario VY Jr, Bersabe MJ, de Leon WU, et al.: Intestinal heterophyidiasis: an emerging food-borne parasitic zoonosis in southern Philippines. Southeast Asian J Trop Med Public Health 2001, 32(Suppl 2):36-42. 